\title{
Evolution of Inequalities in Returns to Education: A Study Based on Tunisian Data
}

Monem abidi ( $\square$ monem.abidi@fsegs.mu.tn )

VPNC Research Laboratory

\section{Research}

Keywords: Yield, education, investment, wage, regression, inequality

Posted Date: December 18th, 2020

DOl: https://doi.org/10.21203/rs.3.rs-129771/v1

License: (c) (i) This work is licensed under a Creative Commons Attribution 4.0 International License. Read Full License 


\title{
EVOLUTION OF INEQUALITIES IN RETURNS TO EDUCATION: A STUDY BASED ON TUNISIAN DATA
}

\author{
Monem Abidi \\ E-mail: monem.abidi@fsegs.rnu.tn \\ VPNC Research Laboratory N-O, FSJEG of Jendouba, University of Jendouba, Tunisia. \\ GSM: (+216)98905017
}

\begin{abstract}
These paper aims are to study and estimate the evolution of the inequalities to the return on educational and professional investment, thus offering vast opportunities for analysis, in particular the dynamic links "profitability" - "investment".

The general view is titled in the research field of human capital theory by analyzing three surveys provided by the National Statistical Institute that focus on wage-employment.

We initially use a corrected Mincer model where we specify two types of regression then we will integrate the indicator of depreciation of human capital. Thus giving results that are used to understand the dynamics of individual investment choices. The results show a decrease in profitability and an increase in catch-up time (Overtaking).

The study also makes it possible to understand the evolution of returns on educational and professional investments. To look for points of failure and imbalances in the education system and its relation to the labor market and to serve as a guide to government policy as it relates to long-term educational programs.
\end{abstract}

J.E.L classification: E24, I21, J24, J31.

Keywords: Yield; education; investment; wage; regression; inequality.

\section{INTRODUCTION}

The formulation of the earnings function of Mincer (1961) is the starting point of a vast literature devoted to the evaluation of returns to education, and which remains the most robust so far. The other advances have tried to take better understand how the present and past environment of an individual affected the returns to education and the differences in the educational choice between individuals synthesized in particular by Card (2001).

The literature that we consulted did not, however, reveal any typology of the consequences of depreciation or the associated models explaining the mutual relationship between the possible effects (Jones, Chonko\& Roberts, 2004; Neuman \& Weiss, 1995; Van Loo, 2005; Shearer \& Steger, 1975; Thijssen, 2005; Lien Laureys, 2014; Zafar Nazarov, NodirAdilov\& Heather LR, 2018).

In this paper, we will try to present the empirical analysis of the earnings model from three investigations, which will be limited, first, to only educational investments. This model is the 
approximation of the general model. Secondly, a presentation of a descriptive statistic will be exposed, and thirdly, the simple schooling model will be extended through the introduction of professional investment. Two types of regression will be presented, which describe the behavior of individuals towards post-school investment. The first is linear, and the second is exponential, in order to measure in a robust way the return on the various educational investments and to detect if there is a depreciation effect or not.

During each step, we will try to compare the different results of different Surveys while respecting the same comparison framework.

The statistical data come from three Surveys made by the INS: the Surveyl done in 1989, which includes after cleaning 7549 people, the Survey2 done in 1999contain5896 people, and the Survey 3 carried out in 2012 which includes 63078 people.

\section{RESULTS AND DISCUSSION}

In terms of results, throughout the study, we tried to draw results beyond very specific formulations and demonstrations that are close to reality.

The analysis makes it possible to compare the output of the estimate based on three job surveys using a new conception of the Mincer model, thus dissecting the factors that influence the decision-making process.

The academic and professional returns thus deduced, make it possible to make comparisons and sketch out the evolution.

The study of the influence of geographic factors and the choice of education pathway allows us to identify disturbing dispersions.

Limits, the main limitation lies in the possibility of adopting instrumental variables (still to prove the validity) for the estimation, which can be the subject of future research under the basis of this new specification with depreciation.

\section{METHODS / EXPERIMENTAL}

This work can be designed to bring value or less in five areas:

Methodologically, where he respects strict scientific standards as a professional in the field, this work oscillates between the traditional guideline and new trends, where we have tried along this paper to be faithful to strict scientific structuring while wearing our specific style and leaving a distinctive imprint on the manuscript

On the knowledge level, the study can be considered as a reference in what relates to the theory of education yield, even that the latter is relatively recent and a little delicate, the present study brings new conceptions while respecting the spirit general of the formulation resulting from the theory. 
On the innovative level, the analysis postulates, to our knowledge, new innovative leads, even if we notice that the approach is addressed to specialists in the field, the reader and from the chain of ideas, can understand the objective as well as the results.

This article reviews the Mincer model, thus specifying a new formulation, centered on the depreciation of the human capital.

The novelty of our reformulation respects the framework of the human capital theory and tries to answer the questions of contemporary society, particularly concerning the return on school and professional investments.

On a practical level, the study is interesting from the point of view that it demonstrates the simplicity and delicacy of the treatment of the return on investment effect while respecting the characteristics of the economy of the employee to be studied, where we took the example of Tunisia.

The study makes it possible to understand the evolution of the returns schools' and professional investments, to look for points of failure and disequilibria in the education system, and the relationship with the labor market. In addition the study can be assessing the impact of government policy concerning educational policies and programs.

\section{PROCESSING OF SURVEY 1}

\subsection{THE SIMPLE SCHOOLING MODEL}

The theoretical study of the different models attached to our analysis shows the robustness of Mincer's modeling, which made it possible to write the natural logarithm of wages as a function of the number of years of study, we will test these two functions:

The theoretical study of the different models attached to our analysis shows the robustness of Mincer's modeling, which made it possible to write the natural logarithm of wages as a function of the number of years of study, we will test these two functions:

$-\log (Y)=\alpha_{0}+\alpha_{1} S+\varepsilon$

$-\log (Y)=\alpha_{0}+\alpha_{1} S+\alpha_{2} S^{2}+\varepsilon$

We can assimilate the coefficient " $\alpha_{0}$ " to "Log $\left(Y_{0}\right) "$, which means that this term expresses the basic minimum wage.

The first regression is in linear form. The independent variable is " $S$ " which expresses the number of years of schooling. The coefficient which is associated with it is " $\alpha_{l}$ " which interpreted as being the average rate of return of educational investments. While " $\log (Y)$ " is the dependent variable, that describes the logarithm of wages.

The introduction of the variable " $S^{2 "}$ in the regression (2) allows testing a parabolic function, and thus sees the shape of the marginal contribution of schooling for different levels of education. The results of the regressions are as follows: 


$$
\log \left(\mathrm{Y}_{1}\right)=3.87+0.0519 S_{1}+\varepsilon \quad R^{2}=19.7 \%
$$

$$
\text { (43.11) }
$$

$$
\log \left(\mathrm{Y}_{1}\right)=3.939+\underset{(0.5355)}{0.0017} S_{1}+\underset{(16.493)}{0.00395} S_{1}^{2}+\varepsilon \quad R^{2}=22.5 \%
$$

Note that the coefficient " $\alpha_{l}$ " is not significant in the regression "2", the average rate of return to schooling is $5.19 \%$, and we note that the coefficient " $\alpha_{2}$ "associated with the square of the education variable is positive, which implies that the return to education increases as the level of education increases. Besides, according to regression "2", we can write:

$$
\frac{\partial \log \left(Y_{1}\right)}{\partial S_{1}}=0,0017+2(0.00395) S_{1}
$$

So for $S_{l}=6$ (primary education) we will have $\tau$ (the yield) $=4.91 \%$

For $S_{1}=9 \quad \Rightarrow \tau=7.28 \%$, for $S_{1}=13 \quad \Rightarrow \tau=10.44 \%$

For $S_{1}=17 \quad \Rightarrow \tau=13.6 \%$

Note that the multiple correlation coefficients are relatively low. The education variable only explains $19.7 \%$ of the first regression and $22.5 \%$ if we introduce the second explanatory variable. This is due to the specification of the simple schooling model.

We can improve the prediction by adopting the catch-up design (Overtaking). The idea is to test a schooling model, the salary of which only corresponds to the school investment, which corresponds in a way to the gross wage at the start of working life. However, the problem is that grosses wages are not directly observable.

We know that the gross salary is higher than the net salary (especially at the start of working life), and we also know that the increase in grosses salary is less than that of the net wage. At some point, the net wage catches up with the initial level of the gross salary, so if we recover this threshold, the estimate of the wage that corresponds to it will be much better.

Most studies estimate that the catch-up period will be between 7 and 9 years of working life. We will test the simple education model, taking special account of this restriction; the number of observations will be reduced to 892 people.

The results are as follows:

$$
\begin{aligned}
& \log \left(\mathrm{Y}_{1}\right)=3.293+\underset{(26.02)}{0.1033} S_{1}+\varepsilon \quad R^{2}=43.21 \% \\
& \log \left(\mathrm{Y}_{1}\right)=3.275+\underset{(5.57)}{0.1082} S_{1}-0.00027 S_{1}^{2}+\varepsilon \quad R^{2}=43.22 \%
\end{aligned}
$$

If we compare these results with those of the previous regression, where we estimated the model using all the observations, we notice that the average rate of return on school investments

\footnotetext{
1 - Student statistics appear in parentheses under the coefficients.

- The index "1" means Surveyl.

- The outputs of the estimation software are available from the author.
} 
doubled from $5.19 \%$ to $10.33 \%$. The coefficient of " $S_{1}^{2}$ " is not significant, which confirms the first approach stating that the return to education increases as the level of education rises.

We also note that the multiple correlation coefficients have improved significantly from $22.5 \%$ to $43.22 \%$, showing that a more accurate measure of school investment allows for more robust estimates.

\subsection{EMPIRICAL ANALYSIS INCLUDING PROFESSIONAL INVESTMENTS}

It is useful to analyze the evolution of wages according to educational background and by age group. To do this, we have grouped the employees in terms of four levels of education:

- None: education equal to zero,

- Primary: from 1 to 6 years of study,

- Secondary: from 7 to 13 years of study,

- Higher: more than 13 years of study.

We distinguish 13 age groups, the extent of each group being four years. The same classification will be adopted when dealing with the wage in terms of the number of years of professional life.

We, therefore, analyze the age-logarithm profiles of wages, and the number of professional years-logarithm profiles of wages (we always calculate the average of the logarithms of wages for each group).

We can write the variation of the logarithm of the net salary for the period " $n$ " and " $n+l$ " in the form:

$$
\begin{aligned}
& \Delta \log Y_{n}=\log W_{n+1}+\log \left(1-\lambda_{n+1}\right)-\log W_{n}-\log \left(1-\lambda_{n}\right) \\
& \log W_{n+1}-\log W_{n}=\log \left(1+\tau \lambda_{n}\right) \phi 0 \\
& \Rightarrow \Delta \log Y_{n}=\log \left(1+\tau \lambda_{n}\right)+\log \left(\frac{1-\lambda_{n+1}}{1-\lambda_{n}}\right)
\end{aligned}
$$

This explains that the variation of the logarithm of the net salary depends on "Log $\left(1+\tau \lambda_{n}\right)$ " and that the concavity is ensured by the decrease of " $\lambda_{n}$ " with age.

The concavity shows that the decrease in " $\lambda_{n}$ " is faster for those with low educational levels than for high levels

- We can see that the decrease in " $\lambda_{n}$ " is common for all groups. However, this decrease is different between the groups; moreover, the crack widens more and more between the first and the second level, while this crack is less significant between the second and the third level.

\footnotetext{
${ }^{2} \lambda_{n}=E_{n} / W_{n}$ the fraction of gross wages allocated to investment.

$W_{n}$ : Gross earnings.

$Y_{n}$ : The net earnings.

$E_{n}$ : Expenses.
} 
- The maximum gain is reached at the end of the career, between 54 and 57 with a delay for the high levels. This is because those who prolong their studies enter with a certain delay in the working life.

Examination of these profiles makes it possible to write:

- With an equal working life, it seems that individuals at the lower level would make much greater investments in the first stage, at the start of working life and up to half of working life. The second stage marks the second half of working life, stamps the boom in investments by people from the upper level, and there will be a widening of the gap between the bottom and the top. Pay inequality, therefore, widens as working life becomes longer.

- It can also be spotted that; the trend curve for the most educated is not concave, which shows that the marginal supplement is not negative, while it is well concave for the least educated. Likewise, the trend line for secondary education is almost linear.

\subsection{CHARACTERIZATION OF THE EQUATIONS TO BE ESTIMATED}

In this section, we will test the gain model taking into account only educational and professional investments, where we define two cases:

- The case without depreciation:

$$
\begin{aligned}
& \log W_{n}=\log E_{0}+\tau_{s} S+\tau_{p} \sum_{i=m}^{n-1} \lambda_{i}+u_{n} \\
& \text { And } \log Y_{n}=\log W_{0}+\tau_{s} S+\tau_{p} \sum_{i=m}^{n-1} \lambda_{i}+\log \left(1-\lambda_{n}\right)+v_{n}
\end{aligned}
$$

- The case with depreciation:

$$
\begin{aligned}
& \log W_{n}=\log W_{0}+\left(\tau_{s}-\gamma\right) S+\sum_{i=m}^{n-1}\left(\tau_{i} \lambda_{i}^{*}-\gamma\right)+u_{n}^{\prime} \\
& =\log W_{0}+\left(\tau_{s}-\gamma\right) S+\tau p \sum_{i=m}^{n-1} \lambda_{i}^{*}-\gamma n+u_{n} \\
& \text { And } \log Y_{n}=\log W_{0}+\left(\tau_{s}-\gamma\right) S+\tau_{p} \sum_{i=m}^{n-1} \lambda_{i}^{*}-\gamma n+\log \left(1-\lambda_{i}^{*}\right)+v_{n}^{\prime}
\end{aligned}
$$

With $W_{n}$ : the gross salary, $Y_{n}$ : the net salary, $S$ : the number of years of schooling, $\tau_{s}$ : the average rate of return on educational investments, $\tau_{p}$ : the average rate of return on professional investments, $\gamma$ : the average rate of depreciation of human capital, $\lambda_{n}$ : the fraction of the salary allocated to professional investment, $\lambda_{n}^{*}$ : the fraction of gross salary allocated to gross investment, $" m "$ : the time of entry into working life, $n$ : the number of years of professional life, and $N$ : the total length of the period of net investment. 
We are going to adopt two regressions that retrace individual behaviors for post-school investment. ${ }^{3}$

The first regression is linear: $\lambda_{n}=\lambda_{0}-\frac{\lambda_{0}}{N} n, " \lambda_{0}$ " explains the part of the gross salary intended for investment during the first period of working life.

The second regression: $\lambda_{n}=\lambda_{0} e^{-\rho n}$ this expression develops the exponential decrease in the part of the salary intended for investment, of parameter " $\rho$ ".

\subsubsection{CHARACTERIZATION WITHOUT DEPRECIATION}

- Using the linear form in the previous model, we will have:

$$
\begin{aligned}
\log _{n} & =\log W_{0}+\tau_{s} S+\tau_{p} \sum_{i=m}^{n-1}\left(\lambda_{0}-\frac{\lambda_{0}}{N} i\right)+u_{n} \\
& =\log W_{0}+\tau_{s} S+\tau_{p} \lambda_{0} n-\frac{\tau_{p} \lambda_{0}}{2 N} n^{2}+u_{n}
\end{aligned}
$$

And $\log Y_{n}=\log W_{0}+\tau_{s} S+\tau_{p} \lambda_{0} n-\frac{\tau_{p} \lambda_{0}}{2 N} n^{2}+\log \left(1-\lambda_{0}+\frac{\lambda_{0}}{N} n\right)+v_{n}$

$\log \left(1-\lambda_{0}+\frac{\lambda_{0}}{N} n\right)=-\left(\lambda_{0}+\frac{\lambda_{0}^{2}}{2}\right)+\left(\frac{\lambda_{0}}{N}+\frac{\lambda_{0}^{2}}{N}\right) n-\frac{\lambda_{0}^{2}}{2 N^{2}} n^{2}$

Taylor's quadratic approximation gives:

Therefore:

$$
\log Y_{n}=\log W_{0}-\lambda_{0}\left(1+\frac{\lambda_{0}}{2}\right)+\tau_{s} S+\left(\tau_{p} \lambda_{0}+\frac{\lambda_{0}}{N}+\frac{\lambda_{0}^{2}}{N}\right) n-\left(\frac{\tau_{p} \lambda_{0}}{2 N}+\frac{\lambda_{0}^{2}}{2 N^{2}}\right) n^{2}+v_{n}
$$

There is a parabolic relationship between the logarithm of wages and the time spent in working life.

- Using the exponential form, the model will be as follows:

$$
\log W_{n}=\log W_{0}+\frac{\tau_{p} \lambda_{0}}{\rho}+\tau_{s} S-\frac{\tau_{p} \lambda_{0}}{\rho} e^{-\rho n}+u_{n}
$$

The expression of the net salary is:

$$
\log Y_{n}=\log W_{0}+\frac{\tau_{p} \lambda_{0}}{\rho}+\tau_{s} S-\frac{\tau_{p} \lambda_{0}}{\rho} e^{-\rho n}+\log \left(1-\lambda_{0} e^{-\rho n}\right)+u_{n}
$$

The same Taylor's quadratic approximation gives:

$$
\log Y_{n}=\log W_{0}+\frac{\tau_{p} \lambda_{0}}{\rho}+\tau_{s} S-\left(\frac{\tau_{p} \lambda_{0}}{\rho}+\lambda_{0}\right) e^{-\rho n}-\frac{\lambda_{0}^{2}}{2} e^{-2 \rho n}+u_{n}
$$

(Function of Gompertz's salary).

${ }^{3}$ The demonstrations that come are specific to the author. 


\subsubsection{CHARACTERIZATION WITH DEPRECIATION}

By introducing the depreciation effect, we will have:

$$
\begin{aligned}
& \lambda_{n}^{*}=\lambda_{0}^{*}-\frac{\lambda_{0}^{*}}{N} n \\
& \lambda_{n}^{*}=\lambda_{0}^{*} e^{-\rho n}
\end{aligned}
$$

Introducing the first investment specification into the gross wage equation gives:

$\log W_{n}=\log W_{0}+\left(\tau_{s}-\gamma\right) S+\left(\tau_{p} \lambda_{0}^{*}-\gamma\right) n-\frac{\tau_{p} \lambda_{0}^{*}}{2 N^{*}} n^{2}+u_{n}$

$$
\log Y_{n}=\log W_{0}-\lambda_{0}^{*}\left(1+\frac{\lambda_{0}^{*}}{2}\right)+\left(\tau_{s}-\gamma\right) S
$$

And

$$
+\left(\tau_{p} \lambda_{0}^{*}+\frac{\lambda_{0}^{*}}{N^{*}}+\frac{\lambda_{0}^{*^{2}}}{N^{*}}-\gamma\right) n-\left(\frac{\tau_{p} \lambda_{0}^{*}}{2 N^{*}}+\frac{\lambda_{0}^{*^{2}}}{2 N^{*^{2}}}\right) n^{2}+v_{n}
$$

The second gives:

$$
\log W_{n}=\log W_{0}+\frac{\tau_{p} \lambda_{0}^{*}}{\rho}+\left(\tau_{s}-\gamma\right) S-\frac{\tau_{p} \lambda_{0}^{*}}{\rho} e^{-\rho n}-\gamma n+u_{n}
$$

We can, therefore, deduct the net salary:

$$
\log Y_{n}=\log W_{0}+\frac{\tau_{p} \lambda_{0}^{*}}{\rho}+\left(\tau_{s}-\gamma\right) S-\left(\frac{\tau_{p} \lambda_{0}^{*}}{\rho}+\lambda_{0}^{*}\right) e^{-\rho n}-\frac{\lambda_{0}^{*^{2}}}{2} e^{-2 \rho n}-\gamma n+u_{n}(4)
$$

For the econometric study, we only use the equations which describe the net salary: (1), (2), (3), (4).We estimate the specifications proceeding in the linear form $((1)$ and (3)) by the following equation:

$\log (Y)=\alpha_{0}+\alpha_{1} S+\alpha_{2} Z+\alpha_{3} Z^{2}+\varepsilon$

Knowing that, $S$ : number of years of study, $Z$ : number of years of professional life, $Z^{2}$ : square of $Z$.

For the specification without depreciation, the coefficients of the equation are defined such that:

$$
\begin{array}{ll}
\alpha_{0}=\log W_{0}-\lambda_{0}\left(1+\frac{\lambda_{0}}{2}\right), & \alpha_{1}=\tau_{s}, \alpha_{2}=\left(\tau_{p} \lambda_{0}+\frac{\lambda_{0}}{N}+\frac{\lambda_{0}^{2}}{N}\right), \\
\alpha_{3}=-\left(\frac{\tau_{p} \lambda_{0}}{2 N}+\frac{\lambda_{0}^{2}}{2 N^{2}}\right) &
\end{array}
$$

And with depreciation: 
$\alpha_{0}=\log W_{0}-\lambda_{0}^{*}\left(1+\frac{\lambda_{0}^{*}}{2}\right), \quad \alpha_{1}=\tau_{s^{-}-\gamma} \quad \alpha_{2}=\left(\tau_{p} \lambda_{0}^{*}+\frac{\lambda_{0}^{*}}{N^{*}}+\frac{\lambda_{0}^{*^{2}}}{N^{*}}\right)-\gamma$

$\alpha_{3}=-\left(\frac{\tau_{p} \lambda_{0}^{*}}{2 N^{*}}+\frac{\lambda_{0}^{*^{2}}}{2 N^{*^{2}}}\right)$

Then, we estimate the specifications proceeding in the exponential form ((2) and (4)) by the following equation:

$\log (Y)=\beta_{0}+\beta_{1} Z+\beta_{2} G+\beta_{3} G^{2}+v$

Knowing that, $G=e^{-\rho n}$ and $G^{2}=e^{-2 \rho n}$

Without depreciation, the coefficients of the equation are defined such that:

$\beta_{0}=\log W_{0}+\frac{\tau_{p} \lambda_{0}}{\rho}, \quad \beta_{1}=\tau_{s}, \quad \beta_{2}=-\left(\frac{\tau_{p} \lambda_{0}}{\rho}+\lambda_{0}\right), \quad \beta_{3}=-\frac{\lambda_{0}^{2}}{2}$.

With depreciation:

$\beta_{0}=\log W_{0}+\frac{\tau_{p} \lambda_{0}^{*}}{\rho}, \quad \beta_{1}=\tau_{s}-\gamma, \quad \beta_{2}=-\left(\frac{\tau_{p} \lambda_{0}^{*}}{\rho}+\lambda_{0}^{*}\right), \quad \beta_{3}=-\frac{\lambda_{0}^{*^{2}}}{2}$,

$\beta_{4}=-\gamma$.

\subsection{RESULT OF THE ESTIMATES}

$\log \left(Y_{1}\right)=\underset{(55)}{.0793} S_{1}+\underset{(24.88)}{0.0397} Z_{1}-\underset{(-17.73)}{0.0005} Z_{1}^{2}+u$

$R^{2}=29.63 \%$.

- We note that all the variables are significant for all the models.

- The introduction of the variable " $Z_{l}$ " in the model improves the explanatory power of the regression, where we note that the multiple correlation coefficients go from $19.7 \%$ to $26.7 \%$.

- Taking into account the variable " $Z_{l}$ " partly eliminates the bias that affected the estimation of the rate of return to education. This bias is explained, on the one hand, by the fact that at the same age the most educated have a shorter professional life, on the other hand, by the increase in the average level of education of the population; which results in a downward biased estimate of the rate of return on school investment.

- The introduction of the variable " $Z_{1}^{2}$ " corrects the average rate of return on school investment, which is around $7.93 \%$ (instead of $5.19 \%$ ).

The calculation of marginal rates of return on school investments makes it possible to write: 
$\frac{\partial \log \left(Y_{1}\right)}{\partial S_{1}}=0.04478+2(0.00247) S_{1}$

This means that for:

$S_{1}=6$ (Primary education) we will have $\tau_{s}$ (The yield) $=7.44 \%$

$\begin{array}{lll}S_{1}=9 & \Rightarrow & \tau_{s}=8.92 \%, \quad S_{l}=13 \Rightarrow \quad \tau_{s}=10.9 \%, \\ S_{1}=17 & \Rightarrow & \tau_{s}=12.87 \% .\end{array}$

There is an increase in yields from all levels, except for the higher level there is a decrease.

It should be noted here that the classic pattern of marginal returns is not respected since they are increasing so that the level of educational attainment increases.

The calculation of the profitability of professional investment goes through the calculation of " $\alpha_{2} "$ and " $\alpha_{3} "$. Besides, we know that:

$$
\begin{aligned}
& \alpha_{2}=\left(\tau_{p} \lambda_{0}+\frac{\lambda_{0}}{N}+\frac{\lambda_{0}^{2}}{N}\right), \quad \alpha_{3}=-\left(\frac{\tau_{p} \lambda_{0}}{2 N}+\frac{\lambda_{0}^{2}}{2 N^{2}}\right), \text { so : } \\
& \lambda_{0}=N \alpha_{2}+2 N^{2} \alpha_{3} \text { and } \tau_{p}=\frac{\alpha_{2}}{N \alpha_{2}+2 N^{2} \alpha_{3}}-\frac{1+N \alpha_{2}+2 N^{2} \alpha_{3}}{N}
\end{aligned}
$$

We also know that " $\lambda_{0}$ " is between zero and " 1 ", which places restrictions on the choice of " $N$ " (the total length of the investment period)

According to regression (2) we obtain the following results:

$$
\text { For } \begin{array}{ll}
N=20 & \Rightarrow \lambda_{0}=39.54 \% \text { and } \tau_{p}=3.08 \%, \\
N=25 & \Rightarrow \lambda_{0}=36.92 \% \text { and } \tau_{p}=5.29 \%, \\
N=30 & \Rightarrow \lambda_{0}=29.31 \% \text { and } \tau_{p}=9.25 \% .
\end{array}
$$

We can, therefore, see the low returns on professional investments in the Tunisian economy. The cumulative duration of professional investments is:

$X_{1}=\sum_{j=0}^{N} \lambda_{j}=\lambda_{0} \frac{N}{2}, \quad$ So $\quad \lambda_{0}=N \alpha_{2}+2 N^{2} \alpha_{3} \Rightarrow X_{1}=\frac{N^{2}}{2} \alpha_{2}-N^{3} \alpha_{3}$, the condition of maximization of $X_{1}$ give $\frac{\partial X_{1}}{\partial N}=0 \quad \Rightarrow N \alpha_{2}-3 N^{2} \alpha_{3}=0$, for $N \neq 0$ we will have $N=\frac{\alpha_{2}}{3 \alpha_{3}}=26.46 \quad \Rightarrow \lambda_{0}=25.08 \% \Rightarrow \tau_{p}=6.61 \%$ and $X_{1}=3.31$

The cumulative duration of on-the-job investments is at its maximum for $N=26.46$; its time equivalent is, therefore, equal to 3.31 . When " $N_{l}$ " is maximum, the fraction of the salary intended for professional investors will be 0.25 ; the rate of return corresponding to this placement is $6.61 \%$. 
Finally, we note that the sign of the interaction variable " $S Z_{I}$ " which is the product of the variable " $S_{I}$ " and the variable " $Z_{l}$ ", is negative which translates a convergence of the profiles of wages and years of professional life.

\subsection{THE RESULTS OF THE GOMPERTZ FUNCTION ESTIMATE}

The advantage of this method is to provide all the information without being forced to resort to other extrinsic information:

$$
\begin{aligned}
& \log \left(Y_{1}\right)=4.018+\underset{(55.55)}{0.0799} S_{1}-\underset{(-8.219)}{0.7405} X_{1}-\underset{(-2.177)}{0.2381} X_{1}^{2}+v \\
& R^{2}=29.45 \%
\end{aligned}
$$

The value taken for " $\rho$ " is 0.0615 (other regressions were made for different values of " $\rho$ ", but we only keep the good one, the one that increases the " $R^{2 "}$ and that the coefficients are significant).

The regression shows that the average profitability of education has not changed (around $7.9 \%)$.

$\beta_{2}=-\left(\frac{\tau_{p} \lambda_{0}}{\rho}+\lambda_{0}\right), \beta_{3}=-\frac{\lambda_{0}^{2}}{2}$, so from " $\beta_{3}$ " we can calculate " $\lambda_{0}$ ", and of " $\beta_{2}$ " we deduce " $\tau_{p} "$, so we will have $\lambda_{0}=69.01 \%$ and $\tau_{p}=0.44 \%$.

The study of depreciation refers to the regression of the model 4 , where the variable " $Z_{l}$ " is introduced (the coefficient of this variable expresses the rate of depreciation of human capital during working life).

Hence $\gamma=0.91 \%$. We note that this coefficient is negative and significant.

So we will have: $\tau_{s}=\beta_{1}+\gamma=0.0799+0.0091=8.9 \%$.

\section{PROCESSING OF SURVEY 2}

\subsection{THE SIMPLE SCHOOLING MODEL}

The estimation of the model gives the following results:

$$
\begin{aligned}
& \log \left(\mathrm{Y}_{2}\right)=4.848+0.0648 S_{2}+\varepsilon \\
& R^{2}=31.6 \% \\
& \log \left(\mathrm{Y}_{2}\right)=5.032-0.0079 S_{2}+0.004499 S_{2}^{2}+\varepsilon \quad R^{2}=34.7 \% \\
& (-1.74) \quad \text { (16.66) }
\end{aligned}
$$

\footnotetext{
4 - Student statistics appear in parentheses under the coefficients.

- The index "2" means Survey2.
} 
The return on education is $6.48 \%$ which is a slight improvement in a decade (5.19\% in 1989). It should be noted that in the second regression the coefficient of " $S_{2}$ " is not significant for a threshold of $5 \%$, but the multiple correlation coefficient is improved, going from $31.6 \%$ to $34.7 \%$.

The calculation of marginal returns gives:

$\frac{\partial \log \left(Y_{2}\right)}{\partial S_{2}}=-0.00079+2(0.00499) S_{2}$

Therefore, we can write:

$S_{2}=6$ (primary education) we will have $\tau_{s}($ the yield $)=5.9 \%$

$S_{2}=9 \quad \Rightarrow \quad \tau_{s}=8.9 \%, \quad S_{2}=13 \Rightarrow \quad \tau_{s}=12.89 \%$,

$S_{2}=17 \quad \Rightarrow \quad \tau_{s}=16.88 \%$.

The comparison of these yields with those of the first survey shows that, on the one hand, the marginal yields are always increasing with the increase in the years of study; on the other hand, there is an improvement in these yields for all levels. However, the largest increase is captured by the top level (from $13.6 \%$ to $16.88 \%$ ).

The estimation of the model, referring to the principle of Overtaking, and always adopted as a catch-up period between 7 and 9 years of professional life allows more solid estimates. Taking into account this specification, the sample will be reduced to 558 people.

The results will be as follows:

$$
\begin{aligned}
& \log \left(\mathrm{Y}_{2}\right)=4.316+\underset{(22.31)}{0.0935} S_{2}+\varepsilon \quad R^{2}=47.24 \% \\
& \log \left(\mathrm{Yl}_{2}\right)=5.056-\underset{(-3.62)}{0.0896} S_{2}+\underset{(7.496)}{0.00926} S_{2}^{2}+\varepsilon \quad R^{2}=52.09 \%
\end{aligned}
$$

These estimates show an improvement in the return to education (from $6.48 \%$ to $9.35 \%$ ), but this increase is less than that recorded at the time of the study of the first survey (from $5.19 \%$ to $10.33 \%$ ), which may indicate a bad signal regarding the contribution of education. But, certainty is far from proven. On the other hand, there is a marked improvement in the level of explanatory power which drops to $52.09 \%$.

\subsection{EMPIRICAL ANALYSIS WITH PROFESSIONAL INVESTMENT}

In this section, we will retain the same approach as that adopted in the study of the Surveyl (four levels of education, thirteen age groups, range of four years, and we calculate the average of the logarithms of wages for each group). 
We not that at the same age we always have the logarithm of the wages of the most educated is higher than that of the less educated. This look is appreciated during working life, especially between primary and secondary level, between secondary and higher level. There are two stages, the first is characterized by a narrowing of the crack between the two levels until the period 38-41, and the second phase marks a divergence. We can also easily see that these profiles are neither flat nor parallel (describing the presence of an investment other than education).

We can see that the concavity is clearer for the low levels than for the higher levels, and the decrease in " $\lambda_{n}$ " is always higher for the lower levels than for the higher levels, which explains the widening of the slot between the gain profiles of the different levels.

Finally, we can locate that the maximum is reached at the end of the career for the upper and secondary level, and a little less for the other two levels.

The investigation brings out the following observations:

- We have two stages of professional investment (with an equal duration of professional life).

The first mark of attempts to catch up on the part of the lower levels, signalled by a greater professional investment; the second stage is the rise of the most educated.

- We can also note that the trend curve for individuals from higher education is almost linear; however this phenomenon was recorded for a lower level during the study of the surveyl. The other curves have the concave shape.

- The most educated individuals have a shorter professional life.

- At the start of a career, salaries are different between the different levels.

\subsection{ESTIMATION OF THE EQUATIONS}

\subsubsection{PARABOLIC FUNCTION}

$$
\log \left(Y_{2}\right)=4.108+\underset{(63.73)}{0.0835} S_{2}+\underset{(26.4)}{0.0425 Z_{2}}-\underset{(-17.22)}{0.0005} 2 Z_{2}^{2}+u
$$

$R^{2}=43.61 \%$.

We can see that all the variables are significant for all the models, except the variables " $Z_{2}$ " and "SZ" in model 2.

It should also be noted that introducing the variable " $Z_{2}$ " improves the explanatory power of the model $(40.78 \%$ instead of $31.6 \%)$, since the introduction of this variable partially eliminates the bias that affected the rate of average return on education. 
Switching to the full model by inserting the square of " $Z_{2}$ " readjusts the average rate of return on educational investment from $6.48 \%$ to $8.35 \%$. Compared with the Surveyl, we can detect a slight improvement over a decade (by $0.42 \%$ ).

The study of marginal returns to schooling brings out (model 4):

$\frac{\partial \log \left(Y_{2}\right)}{\partial S_{2}}=0.0391+2(0.00274) S_{2}$

So, we can write:

$S_{2}=6$ (primary education) we will have, $\tau_{s}($ the yield $)=7.2 \%$

$S_{2}=9 \quad \Rightarrow \quad \tau_{s}=8.84 \%, \quad S_{2}=13 \Rightarrow \quad \tau_{s}=11.03 \%$,

$S_{2}=17 \quad \Rightarrow \quad \tau_{s}=13.23 \%$.

There is an increase in marginal yields compared to the simple model, except for the higher level. But, it can be pointed out when comparing the two surveys, that the marginal yields of the lower levels proclaimed a decrease during these 10 years, then they could have increased for the higher levels (from $12.87 \%$ to $13.23 \%$ ), implying a tendency towards the valuation of the high levels to the detriment of those of the low level. But this trend remains weak.

Regarding professional profitability and regarding model "2" gives:

$$
\text { For } \begin{array}{ll}
N=20 & \Rightarrow \lambda_{0}=43.07 \% \text { and } \tau_{p}=2.72 \%, \\
N=25 & \Rightarrow \lambda_{0}=40.72 \% \text { and } \tau_{p}=4.81 \%, \\
N=30 & \Rightarrow \lambda_{0}=33.12 \% \text { and } \tau_{p}=8.4 \% .
\end{array}
$$

We can conclude that at " $N$ " equal, the " $\lambda_{0}$ " has increased since the Surveyl, causing the agents to have tension increased their post-school investments, but we note, on the other hand, that the profitability has decreased. So over the past decade, people have invested more and made less.

According to model 2, the interaction variable " $\mathrm{SZ}_{2}$ " is not significant, which does not allow us to distinguish the relationship between education and experience.

The cumulative duration of post-school investments is:

$$
\begin{aligned}
& X_{2}=\sum_{j=0}^{N} \lambda_{j}=\lambda_{0} \frac{N}{2} \Rightarrow X_{2}=\frac{N^{2}}{2} \alpha_{2}-N^{3} \alpha_{3}, X_{2} \text { is maximum for } N=\frac{\alpha_{2}}{3 \alpha_{3}}=27.01 \quad \Rightarrow \lambda_{0}= \\
& 38.28 \% \Rightarrow \tau_{p}=5.98 \% \text { and } \quad X_{2}=5.16 .
\end{aligned}
$$

The cumulative duration of professional investments reaches its maximum for $X=27.01$; its time equivalent is, therefore, equal to 5.16. If " $X_{2}$ " is maximum, the fraction of the salary spent on onthe-job investment will be 0.382 . The rate of return equivalent to these expenses is $5.98 \%$. 
Comparison of these figures with those of 1989 shows that there is an increase in "N", " $\lambda_{0}$ " and " $X$ " but a decrease in " $\tau_{p} "$.

\subsubsection{THE GOMPERTZ FUNCTION ESTIMATE}

$$
\log \left(Y_{2}\right)=4.991+\underset{(69.91)}{0.08417} S_{2} \underset{(-8.25)}{-0.7875 G_{2}}-\underset{(-2.76)}{0.3061 G_{2}^{2}+v}
$$

$R^{2}=43.86 \%$.

It can be noted that there is no change in the profitability of education, if we compare this model with it from the basic model, using parabolic regression. Similarly, the difference observed is always the same if we compare the figures from the two surveys.

The chosen value of " $\rho$ " this time is 0.066 , so $\lambda_{0}=78.24 \%$ and $\tau_{p}=0.0427 \%$. Similarly, here we can compare the state of an increase in professional investment, while their profitability decreases if we compare the results of the surveys, under the Gompertz regression.

Regarding the state of depreciation, we can refer to the coefficient of the variable " $Z_{2}$ " in the model 4, where we record $\gamma=1 \%$, the coefficient is negative and significant; therefore, the phenomenon of the depreciation of human capital during working life is justified. Moreover, we note that this rate has increased compared to the Surveyl.

The gross profitability of education is, therefore:

$\tau_{s}=\beta_{1}+\gamma=0.08417+0.01=9.41 \%$.

\section{PROCESSING OF SURVEY 3}

\subsection{THE SIMPLE SCHOOLING MODEL}

The estimate shows the following results:

$$
\begin{aligned}
& \log \left(\mathrm{Y}_{3}\right)=5.255+\underset{(143.66)}{0.0598} S_{3}+\varepsilon \quad R^{2}=24.65 \% \\
& \log \left(\mathrm{Y}_{3}\right)=5.568-0.02497 S_{3}+0.004279 S_{3}^{2}+\varepsilon \quad R^{2}=27.61 \%
\end{aligned}
$$

We noted that the average return on education has deteriorated to $5.98 \%$ (against $6.48 \%$ in 1999). The passage to the calculation of marginal returns allows writing:

$$
\frac{\partial \log \left(Y_{3}\right)}{\partial S_{3}}=-0.02497+2(0.004279) S_{3}
$$

\footnotetext{
5 - Student statistics appear in parentheses under the coefficients.

- The index "3" means Survey3.
} 
And therefore:

$S_{3}=6$ (primary education) we will have $\tau_{s}$ (the yield) $=2.63 \%$

$S_{3}=9 \quad \Rightarrow \quad \tau_{s}=5.2 \%, \quad S_{3}=13 \quad \Rightarrow \quad \tau_{s}=8.63 \%$,

$S_{3}=17 \quad \Rightarrow \quad \tau_{s}=12.05 \%$.

First, we can discover that the marginal returns are always increasing with the number of years of study. However, the review of these yields with those of the Survey2, shows a decrease in these yields for all levels, primary, secondary, and higher (degradation by two points for each level) ${ }^{6}$.

Estimating the model by limiting itself to a professional life between 7 and 9 years (Overtaking) reduces the sample to 5,454 people, which gives the following results:

$$
\begin{array}{ll}
\log \left(\mathrm{Y}_{3}\right)=4.56+\underset{(52.22)}{0.098} S_{3}+\varepsilon & R^{2}=33.34 \% \\
\log \left(\mathrm{Y}_{3}\right)=5.96-\underset{(-15.76)}{0.168} S_{3}+\underset{0.0111}{0.25 .3)} S_{3}^{2}+\varepsilon & R^{2}=40.35 \%
\end{array}
$$

Examination of these results shows an improvement in the average return on education ranging from $5.98 \%$ to $9.8 \%$, almost the same rate recorded for the Survey2. But it still remains below the return on education for Surveyl (under the same conditions). However, there is a marked softening in terms of the explanatory power of the model.

\subsection{EMPIRICAL ANALYSIS INCLUDING PROFESSIONAL INVESTMENTS}

In this section, we will use the same grouping procedure as during the processing of the two previous surveys, to allow reliable comparisons between the different surveys.

The first observation to be made is that which is similar to the pattern of earnings profiles, where there is a marked improvement compared to the two previous surveys.

At the start of working life, the profiles are almost the same except for that of the superior. Then they will be well distinguished towards the end of working life. However, these profiles were well distinguished from the start for the other two surveys.

At the same age, the same principle prevails, the most educated people on the higher profiles than the least educated. And this pattern will be sustained during active life with an increasing and continuous rhythm, the possibilities of catching up for low levels of which are almost zero (attempts recorded during the study of Surveys1 and 2).

${ }^{6}$ The comparison with Surveyl shows a decrease in marginal returns by one point for each level of education. 
The concavities of the profiles in this survey are checked for all levels of training. The concavity will be compared to the decrease in " $\lambda_{n}$ " as the age increases; this decrease is more acute for the low levels.

The logarithm of wages peaks when workers cross the 54-57 age range. It can be seen that the most educated have a shorter professional life than the others. We note also that the most educated allocate larger sums of money to professional investment, which always confirms the positive correlation between school and post-school investments, but the comparison with the two other surveys shows that the most educated spend less and less on professional investment. This is explained by the decreasing return over time of this type of investment.

\subsection{ESTIMATION OF THE EQUATIONS}

\subsubsection{PARABOLIC FORM}

The estimation of the model gives:

$$
\begin{aligned}
& \log \left(Y_{3}\right)=4.433+\underset{(188.09)}{0.0834} S_{3}+\underset{(82.07)}{0.04109} Z_{3}-\underset{(-45.05)}{0.000488} Z_{3}^{2}+u \\
& R^{2}=39.67 \%
\end{aligned}
$$

The study of the outputs of the estimates shows that all the variables are significant, we also note that the insertion of the variable " $Z_{3}$ " and " $Z_{3}{ }^{2 n}$ increases the explanatory power of the model $(39.67 \%)$ and the average yield of the educational investment which goes from $5.98 \%$ to $8.34 \%$.

The comparison of these rates with those of the Survey2 indicates a slight decrease in the average profitability of education, which confirms the doubts already noted since the Survey2 concerning the valuation of educational investments.

$$
\begin{aligned}
& \text { Marginal returns to education are calculated from the model2: } \\
& \frac{\partial \log \left(Y_{3}\right)}{\partial S_{3}}=-0.0125+2(0.0047) S_{3}
\end{aligned}
$$

The marginal returns from the different levels of education are thus:

$S_{3}=6$ (primary education) gives $\tau_{s}($ the yield $)=4.39 \%$

$$
\begin{array}{lll}
S_{3}=9 & \Rightarrow & \tau_{s}=7.22 \%, \\
S_{3}=13 & \Rightarrow & \tau_{s}=10.99 \%, \\
S_{3}=17 & \Rightarrow & \tau_{s}=14.75 \% .
\end{array}
$$

We note that there is a growth in marginal returns compared to the simple schooling model, almost at the rate of two points for each level of education. The comparison of these results with that 
of the Survey2 reveals a decrease in marginal returns for all levels of education, except for the case $S_{3}$ $=17$, and the fact observed during the study of the Survey 2 is proven in this Survey 3.

In terms of the profitability of professional investments, we can calculate for different values of " $N$ ", from model 2:

$$
\begin{array}{cll}
\text { For } N=20 & \Rightarrow \lambda_{0}=43.1 \% & \text { and } \tau_{p}=2.38 \%, \\
N=25 & \Rightarrow \lambda_{0}=41.66 \% & \text { and } \tau_{p}=4.19 \%, \\
N=30 & \Rightarrow \lambda_{0}=35.33 \% & \text { and } \tau_{p}=7.11 \% .
\end{array}
$$

If we compare the results of the three surveys, we notice that for " $N$ " equal, " $\lambda_{0}$ " has been increasing since the 1989survey, which shows that people are attaching more and more important to the post-school investment. However, profitability continues to decline.

The interaction variable " $S Z_{3}$ " has a positive and significant coefficient, which indicates that there is a complementary relationship between formal education and post-school training.

The cumulative duration of professional investments is:

$$
\begin{aligned}
& X_{3}=\sum_{j=0}^{N} \lambda_{j}=\lambda_{0} \frac{N}{2} \Rightarrow X_{3}=\frac{N^{2}}{2} \alpha_{2}-N^{3} \alpha_{3}, X_{3} \text { is maximum for } N=\frac{\alpha_{2}}{3 \alpha_{3}}=28.03 \quad \Rightarrow \lambda_{0}= \\
& 38.4 \% \Rightarrow \tau_{p}=5.76 \% \text { and } \quad X_{3}=5.38 .
\end{aligned}
$$

The maximum of the cumulative duration of professional investments is reached for " $N=$ 28.03 " for a time equivalent " $X_{3}=5.38$ ", the part of the salary allocated to the investment professional is therefore 0.384 , with a rate of return equal to $5.76 \%$.

We note that the achievement of the optimum, and by comparing it with the figures found during the processing of the Surveyl and 2 is characterized by an increase in the investment period " $N$ ", the share of income spent on investment " $\lambda_{0}$ " and of " $X$ ", on the other hand, there is a decrease in profitability " $\tau_{p}$. This builds contradictions in the economy.

\subsubsection{GOMPERTZ FUNCTION ESTIMATE}

$$
\log \left(Y_{3}\right)=5.372+\underset{(194.25)}{0.0836} S_{3}-\underset{(-30.55)}{0.944} G_{3}-\underset{(-2.71)}{0.0832} G_{3}^{2}+v
$$

$R^{2}=39.88 \%$. (Appendix 3.2, model 4).

We note that the explanatory power of the model, as well as the return to schooling, did not change compared to the initial model with parabolic regression. However, the return to average education is estimated to decrease compared to the Survey2 under the Gompretz specification. 
In this case, the value of " $\rho$ " chosen is equal to 0.058 . We can, therefore, notice that the " $\rho$ " chosen for the three surveys is around $6 \%$.

So, $\lambda_{0}=40.8 \%$ and $\tau_{p}=7.62 \%$. Consequently, it can be said that this generation benefited from the benefits of professional investments from previous generations since the proportion " $\lambda_{0}$ " devoted to post-school investment decreases while profitability increases compared to the Surveyl and 2 in part of this model.

The inclusion of the variable " $Z_{3}$ " in model 5 makes it possible to deduce the value of the depreciation coefficient $\gamma=1.27 \%$, which is negatively significant. So, there, once again, the phenomenon of the depreciation of human capital is justified. Moreover, it has been increasing since the Surveyl.

We can, therefore, deduct the average return on education without depreciation (gross return): $\tau_{s}=\beta_{1}+\gamma=0.0836+0.01279=9.64 \%$.

If we compare the gross return on the education of the three surveys, we can see a slight increase ranging from $8.9 \%$ in 1989 to $9.41 \%$ in 1999 to stabilize at $9.64 \%$ in 2012.

\section{CONCLUSION}

The construction of a gain function inspired by the $H C$ models made it possible to apprehend satisfactorily the returns on investment in training, by overcoming the shortcomings noted.

We have seen in this paper, the analysis of wage formation through three surveys. We were able to identify, during the processing of the 1989survey, which the average internal return on education in the simple schooling model is $5.16 \%$, whereas it is $10.33 \%$ if we make use of catch-up design, to reach $7.93 \%$ in the full model with post-school investment. Estimating the Gompertz function also gives similar results, yielding 7.99\%.

Concerning the 1999 survey, the simple education model gives a return of $6.48 \%$ and with the conditions of catch-up amounts to $9.35 \%$, while it is $8.35 \%$ in the model including professional investments. Likewise, the Gompertz function estimate confirms the complete model with a rate of return of $8.41 \%$.

The examination of the 2012 survey shows a rate of return of $5.98 \%$ in the simple model. The catch-up specification amounts to $9.8 \%$, while it is $8.31 \%$ in the case of the addition of investment on the job, a rate which is confirmed by Gomertz's estimate which presents a rate of $8.36 \%$. 
A comparison between these different rates under the different conditions shows that, overall, the internal average return on education is deteriorating.

Besides, the study of the dynamics of marginal returns to education also shows depreciation for all levels of education.

Regarding the profitability of professional investments, we note that since 1989 , the share of income devoted to professional investment has been constantly increasing, while profitability has been constantly decreasing, so we are also witnessing depreciation in professional investment.

\section{Conflicts of interest}

The author declares that this article does not include any conflicts of interest, either potential or apparent.

\section{Declarations}

* Ethics approval and consent to participate: Not applicable

* Consent for publication: Not applicable

* Availability of data and material: The data supporting the results of this study are available from [National Institute of Statistics (NIS)] but restrictions apply to the availability of these data, which have been used under license for this study, and are therefore not accessible to the public. Data are, however, available from the authors upon reasonable request and with permission from [NIS].

* Competing interests: Not applicable

* Funding: Not applicable

* Authors' contributions: All authors have read and approved the final manuscript

* Acknowledgements: Not applicable

* Authors' information: Not applicable

\section{List of abbreviations}

$\log \left(Y_{0}\right)=\alpha_{0}$ : The basic minimum wage.

$S$ : The number of years of schooling.

$S^{2}:$ Square of $S$.

$\alpha_{1}$ : The average rate of return of educational investments.

$\log (Y)$ : the logarithm of wages.

$\tau:$ The yield.

$\lambda_{\mathrm{n}}$ : The fraction of gross wages allocated to investment.

$W_{n}$ : Gross earnings. 
$Y_{n}$ : The net earnings.

$E_{n}$ : Expenses.

$W_{n}$ : The gross salary.

$Y_{n}$ : The net salary.

$\tau_{s}$ : The average rate of return on educational investments.

$\tau_{p}$ : The average rate of return on professional investments.

$\gamma:$ the average rate of depreciation of human capital.

$\lambda_{n}$ : The fraction of the salary allocated to professional investment.

$\lambda_{n}^{*}:$ The fraction of gross salary allocated to gross investment.

$m$ : the time of entry into working life.

$n$ : The number of years of professional life.

$N$ : The total length of the period of net investment.

$\rho$ : The part of the salary intended for investment.

$G=e^{-\rho n}$ : Specifications proceeding in the exponential form.

$Z$ : Number of years of professional life.

$Z^{2}$ : Square of $Z$.

$X_{1:}:$ The cumulative duration of professional investments 


\section{REFERENCES}

Akerlof, G."Employer LearningAnd Statistical Discrimination."Quarterly Journal Of Economics, 116(2001): 313-350.

Altonj, I.; Pierret, C.“Employer Learning Statistical Discrimination." Quarterly Journal Of Economics, 116, (2001): 313-350.

Angrist, J.-D.; Krueger, A.-B."Does Compulsory School Attendance Affect Schooling And Earnings? ."Quarterly Journal Of Economics, 106(1991): 979-1014.

Ashenfelter, O.; Card, D. "Using The Longitudinal Structure Of Earnings To Estimate The Effect Of Training Programs."Review Of Economics And Statistics, (1985): 648-660.

Ashenfelter, O.; Kruger, A."Estimates Of The Economic Return To Schooling From A New Sample Of Twins."American Economic Review, 84(1994): 1157-1173.

Barnichon, R.; Andrew, F. "Labor Market Heterogeneity And The Aggregate Matching Function: Dataset."American Economic Journal: Macroeconomics, 7(4)(2015): 222-249.

Barro, R.J.; Lee, J.W.“International Measures Of Schooling Years And Schooling.”American Economic Review, №86, Vol 2(1996):218-223.

Becker, G.; Chiswick, B.“Education And The Distribution Of Earnings.”American Economic Review, (1966) :Mai.

Beggs, J.-J.; Chapman, B.-J.“Labor Turnover Bias In Estimating Wages.”Review Of Economics And Statistics, 70(1988): 117-123.

Bishop, J.H.;Wöbmann, L."Institutional Effects In A Simple Model Of Educational Production."Keil, Working Paper,(2001): º1085.

Boissiere, M.; Knight, J.-B.; Sabot, R.-H.“Earnings, Schooling, Ability And Cognitive Skills.”American Economic Review, 75(1985): 1016-1030.

Brodaty, T.O.; Gary-Bobo, R.J.; Prieto, A."Do Risk Aversion And Wages Explain Educational Choices."Journal Of Public Economics, Vol. 117(2014): 125-148.

Falaris, E.-M.; Peters, H.-E."Survey Attribution And Schooling Choices.”Journal Of Human Resources, 33(1998): 531-554.

Fitzgerald, J.;Gottschack, P.;Mofftt,R.."The Impact Of Attrition The Panel Study Of Income Dynamics On Intergenerational Analysis."Journal Of Human Resources, 33(1998): 300-344.

Harmon, C.; Walker, I.“Estimates Of The Economic Return To Schooling For The Uk.”American Economic Review, 85(1995):1278-1286.

Hungerford, T.; Solon, G.“Sheep Skin Effects In The Returns To Education.”Review Of Economics And Statistics, 69 (1987):175-177.

Hwang, H.; Reed, W.-R.; Hubbard, C.“Compensating Wage Differentials And Unobserved Productivity."Journal Of Political Economic, 100(1992): 835-858.

Mincer, J."Schooling, Experience, And Earnings." National Bureau Of Economic Research. (1974). 
Riley, J. “Information, Screening And Human Capital."American Economic Review, 66, (1976): 254-260.

Shimer, R."Reassessing The Ins And Outs Of Unemployment."Review Of Economic Dynamics, 15 (2)(2012): 127-48.

Stiglittz, J. "The Theory Of Screening, Education And The Distribution Of Income."American Economic Review, 65 (1975): 283-300.

Zafar, N.;Nodir, A.;Heather, L-R. "Human Capital Depreciation AndStigma Effects In Unemployed Workers' Re- Employment Wages.”In SSRN Electronic Journal(2018)DOI: 10.2139/Ssrn.3223708. 


\section{Supplementary Files}

This is a list of supplementary files associated with this preprint. Click to download.

- Theoutputofsoftwarefordifferentestimates.docx 